\title{
OPTIMIZACIÓN DE PROCESOS MARKOVIANOS DE DECISIÓN A TRAVÉS DE UN MODELO DE PROGRAMACIÓN LINEAL: EL CASO DE INVERSIÓN EN ACTIVOS FINANCIEROS RIESGOSOS
}

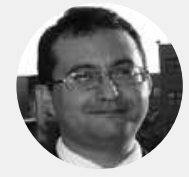

\author{
ADOLFO G. BOUILLON
}

- Profesor de Administración de Operaciones

Área Académica de Administración

Facultad de Negocios, UPC

RESUMEN

El presente artículo busca determinar un curso de acción óptimo para la inversión en activos riesgosos, a través de un modelo de programación lineal adaptado a un tipo particular de decisiones, conocido como proceso markoviano de decisión. Para ello se analizan las estadísticas de las acciones de tres empresas importantes en tres industrias de amplia representatividad bursátil y su correlación con el Indice General de la Bolsa de Valores de Lima, con el fin de establecer un modelo de predicción de los precios de dichos activos, a partir de lo cual se desarrolla una formulación de toma de decisiones con el proceso estadístico antes mencionado. Finalmente, se prepara un modelo de programación lineal para llegar a la solución óptima. Se concluye que el modelo de programación lineal ayuda a reducir considerablemente el tiempo y la complejidad de un proceso markoviano de decisión al momento de elegir un curso de acción que maximice la rentabilidad de invertir en activos riesgosos.

Palabras clave: proceso de decisión markoviano, programación lineal, toma de decisiones en inversiones financieras.

\section{ABSTRACT}

This paper seeks to determine an optimal course of action for investing in risky assets through a linear programming model, adapted to a particular type of decision, known as the Markovian decision process. We analyze stock statistics of three important firms of three industries of representative trading activity and their correlation with the Lima Stock Exchange General Index, with the purpose of establishing a prediction price model for said assets.

Then, we formulate a Markovian decision process and prepare a linear programming model for an optimal solution. We find that linear programming modeling reduces the time and complexity of a Markovian decision process in choosing a course of action that maximizes profits upon risky asset investment.
Keywords: Markovian decision process, linear programming, financial investment decision-making

El objetivo de todo sistema de gestión es tomar el mejor curso de acción, dados los recursos y el ambiente en el que se desarrolla la empresa sobre la que se toma dicha decisión. Desde el enfoque de la teoría general de sistemas, el objetivo es optimizar una variable de interés del sistema, que permita mejorar su desempeño hacia el futuro.

El uso del método científico en la toma de decisiones abre todo un campo en la administración, conocido en inglés como management science, y que viene desarrollando herramientas para tal fin. En ese sentido, los procesos estadísticos conocidos como estocásticos son de gran utilidad, pues utilizan el concepto de la probabilidad, asociado a la posibilidad de que un evento ocurra en la vida real. Uno de estos tipos de procesos se conoce como "cadena de Markov"; y la toma de decisiones en dichas estructuras se conoce, por ende, como procesos markovianos de decisión (PMD).

En este proceso de búsqueda de alternativas para tomar decisiones adecuadas, se tienen los modelos matemáticos, que buscan representar de forma adaptada la realidad y facilitan dicho proceso. Uno de los modelos matemáticos determinísticos más utilizados es la programación lineal, que considera las restricciones de recursos con los que los sistemas cuentan para lidiar con la realidad y tratar de optimizarla.

En el presente artículo se desarrolla un modelo de programación lineal para encontrar un curso de acción en el problema de decidir en qué activo riesgoso invertir. Este proceso de decisión en inversión de acciones se modela como un proceso markoviano de decisión. Para tal fin, se evalúa el comportamiento de tres acciones de industrias representativas, seleccionadas de la Bolsa de Valores de Lima y su correlación con el indicador del mercado en la plaza peruana. Posteriormente, se presenta el modelo de decisión markoviano a partir de la obtención de la matriz de probabilidades de transición, para finalmente desarollar y presentar el modelo de programación lineal que entregará la política óptima de inversión. 


\section{PROCESOS MARKOVIANOS DE DECISIÓN}

Los procesos de decisión markovianos son aquellos que poseen la propiedad "markoviana". Una manera de introducir este tema es comentar el origen del mismo. Una cadena de Markov es un proceso estocástico, es decir, un proceso cuyos estados se van presentando a través de probabilidades de transición, desde un estado $i$ a un estado $j$. Respecto a la propiedad markoviana, ésta se refiere a que la probabilidad de pasar del estado $i$ al estado j no depende de lo que haya sucedido antes de llegar al estado i. Así, se puede describir, a largo plazo, el estado estable del proceso, es decir, conocer las probabilidades de cada estado a largo plazo.

Sin embargo, el modelo se puede volver dinámico, en el sentido de que se pueden tomar decisiones respecto a cada estado del sistema, pudiendo alterar no sólo las probabilidades de transición, sino también el costo o el beneficio esperado de largo plazo del tomador de decisiones.

De esta manera, un proceso de decisión markoviano puede estar estructurado de la siguiente manera (Hillier, 2010): i) se tiene un número identificado de estados $i$ en el proceso estocástico; ii) se tiene un conjunto de decisiones $k$, que se pueden tomar en cada estado i; iii) cuando se toma una decisión, se puede afectar la probabilidad de transición al siguiente estado; iv) el conjunto de decisiones que se va tomando en cada estado se considera una política; v) el objetivo del proceso de decisión es encontrar una política óptima que minimice (maximice) el costo (beneficio) esperado a largo plazo.

Justamente el desarrollo del tema del presente artículo está centrado en la metodología para encontrar la política óptima; y una interesante opción es el desarrollo del modelo de programación lineal para encontrar dicha política.

\section{PROGRAMACIÓN LINEAL}

La programación lineal se puede entender como una importante herramienta que busca optimizar la asignación de recurso entre diferentes actividades que compiten entre sí por dichos recursos (Taha, 2012). Así, un modelo de programación lineal consta de variables de decisión, que son aquellas que permitirán elegir el curso de acción adecuado. Asimismo, tiene una función objetivo, que busca optimizar una variable de interés, como puede ser la utilidad total o el costo total; y finalmente, tiene una serie de restricciones, que establecen el conjunto de posibilidades de decisión y que están determinadas por los recursos con los que el sistema cuenta.

Existen diferentes técnicas de estos modelos de programación lineal. Sin embargo, el algoritmo más utilizado es el método Símplex. Este consiste en desarrollar una serie de tablas que van encontrando los valores de las variables de decisión que optimizan la función objetivo, utilizando álgebra matricial.
Los campos de aplicación de esta herramienta son bastante amplios; y el ámbito financiero no es la excepción. De hecho, Wei (2016) propone un modelo de decisión de largo plazo que es sensible al riesgo, siendo éste una variable de interés para desarrollar futuras investigaciones en la aplicación de esta técnica de programación, tanto lineal, como no lineal.

\section{PLANTEAMIENTO DEL MODELO}

\section{ELEMENTOS INICIALES}

El desarrollo de la investigación está relacionada a la aplicación de un modelo de programación lineal, como forma de obtener una política óptima de toma de decisiones en inversión de activos riesgosos. Para esto, se trabaja con datos recopilados de diferentes acciones de la Bolsa de Valores de Lima (BVL), a fin de tener la información necesaria respecto de las fluctuaciones bursátiles en general, así como de las acciones seleccionadas, tratando de seleccionar acciones representativas de algunas de las principales industrias locales.

Para lo anterior, tenemos la posibilidad de invertir en una serie de acciones de la plaza limeña; las posibilidades de invertir son por acciones; y se espera un máximo retorno, en función del movimiento del mercado, éste medido por IGBVL. La información se ha recopilado desde el año 2015 hasta mayo de 2018 y se muestra en la Figura 1.

Figura 1. Valores del S\&P/BVL Peru General Index (PEN)

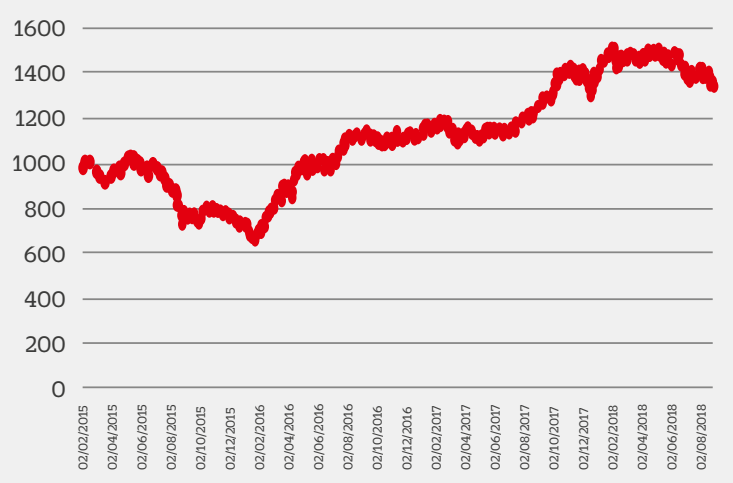

Nota. Adaptado de Bolsa de Valores de Lima [BVL] (2018).

Las acciones seleccionadas para este trabajo son las siguientes:

- Alicorp (ALICORC1)

- Banco de Crédito (CREDITC1)

- Buenaventura (BUENAVC1)

Asimismo, se ha buscado la relación que existe entre el movimiento del mercado y la variación en la rentabilidad de las acciones, de tal forma que podamos tomar una decisión en base a las fluctuaciones que tenga la BVL. Dicha relación se presenta por empresa en las siguientes tres figuras: 
Figura 2. Relación entre Precio por Acción de Alicorp y el IGBVL (2015 - 2018)

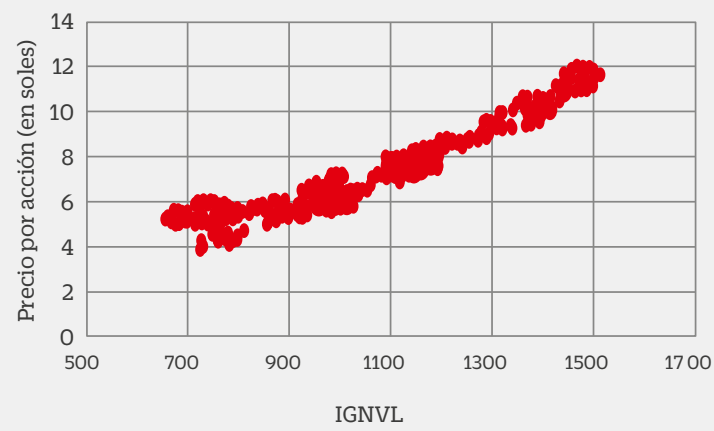

Nota. Adaptado de Bolsa de Valores de Lima [BVL] (2018).

Figura 3. Relación entre Precio por Acción de Banco de Crédito y el IGBVL (2015 - 2018)

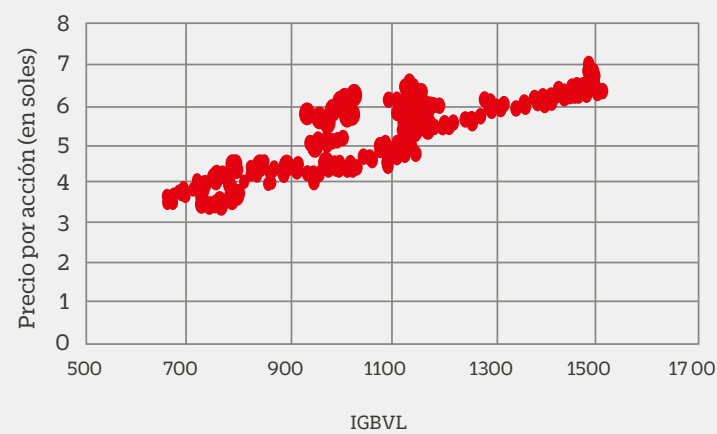

Nota. Adaptado de Bolsa de Valores de Lima [BVL] (2018).

Figura 4. Relación entre Precio por Acción de Buenaventura y el IGBVL (2015 - 2018)

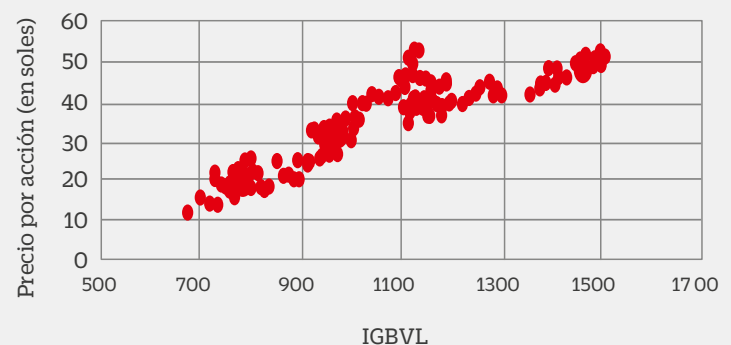

Nota. Adaptado de Bolsa de Valores de Lima [BVL] (2018).

De las figuras 2, 3 y 4, se puede apreciar que existe una correlación positiva entre los precios por acción y el IGBVL. Ello puede hacer aún más difícil la toma de decisiones, debido a que el precio de las acciones sube al aumentar el IGBVL. Entonces ¿en qué acción se debe invertir?

Para efectos del modelo, se debe formalizar una relación de causalidad entre el precio de las acciones y el IGBVL a través del modelo de regresión lineal, utilizando el método de mínimos cuadrados ordinarios (MCO) para poder estimar el precio por acción esperado en función del valor de la variable BVL. La ecuación de regresión tiene la siguiente expresión: $y=\beta_{0}+\beta_{1} x+e$

donde $\mathrm{y}$ = Precio de la acción; $\mathrm{x}$ = Valor del IGBVL .

En las siguientes tablas se muestra la estimación de las ecuaciones de regresión por cada variable seleccionada.

Tabla 1. Resultados del Modelo de Regresión Lineal para las Acciones Seleccionadas

\begin{tabular}{|c|c|c|c|}
\hline $\begin{array}{c}\text { Estadísticas / } \\
\text { Variables }\end{array}$ & ALICORC1 & CREDITC1 & BUENAVC1 \\
\hline $\begin{array}{c}\text { Coeficiente de } \\
\text { determinación } \mathrm{R}^{2}\end{array}$ & 0.9252 & 0.6794 & 0.8281 \\
\hline Coeficiente $\beta_{0}$ & -1.8159 & 1.6354 & -9.5699 \\
\hline$p$-value & 0.0000 & 0.0000 & 0.0000 \\
\hline Coeficiente $\beta_{1}$ & 0.0086 & 0.0033 & 0.0420 \\
\hline$p$-value & 0.0000 & 0.0000 & 0.0000 \\
\hline
\end{tabular}

De la información presentada en la Tabla 1 se puede afirmar que todos los coeficientes de las ecuaciones de regresión son significativos al 95\% de confianza. Asimismo, los coeficientes de determinación superan el 80\% para las acciones Alicorp y Buenaventura, mientras que para el Banco de Crédito es 68\%. Con estas ecuaciones se podrá conocer el valor del precio de las acciones cuando la variable IGBVL cambie de estado.

\section{CONFIGURACIÓN DEL PROCESO DE DECISIÓN MARKOVIANO (PDM)}

De esta manera, tenemos el siguiente problema que se puede modelar a través de una cadena de Markov.

\section{ESTADOS DE LA NATURALEZA}

Considerando las diferentes variaciones que tiene el IGBVL apreciadas en la Figura 1, se ha propuesto establecer intervalos, con la finalidad de identificar los estados de la variable de interés.

Tomando en cuenta que en los últimos periodos el índice se ha consolidado en valores mayores a los 1,400 puntos, se ha considerado los valores desde enero de 2018 hasta mayo de 2018, totalizando 104 observaciones. Las estadísticas descriptivas se registran en la Tabla 2, a continuación:

Tabla 2. Principales Estadísticas Descriptivas de la Variable IGBVL

\begin{tabular}{|c|c|}
\hline Indicador & Valor \\
\hline Media & $1,470.88$ \\
\hline Desviación Estándar & 17.88 \\
\hline Rango & 83.79 \\
\hline Mínimo & $1,427.23$ \\
\hline Máximo & $1,511.02$ \\
\hline Cuenta & 1,004 \\
\hline
\end{tabular}


Figura 5. Evolución del Î́ndice IGBVL en el Año 2018

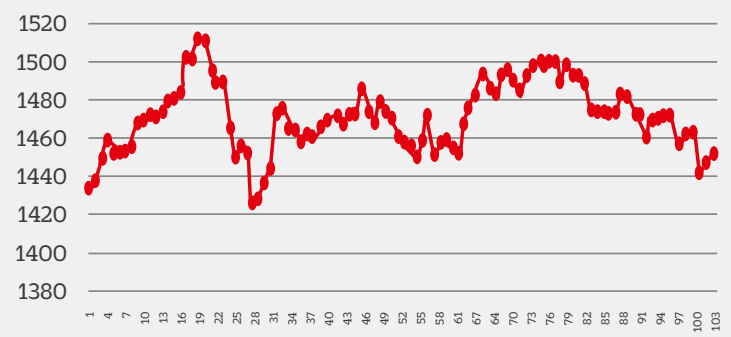

Nota. Adaptado de Bolsa de Valores de Lima [BVL] (2018).

Tomando en cuenta el rango de 83.79 y el número de estados propuestos, que en este caso son cinco, se obtiene un ancho de clase de 16.76 puntos. De esta manera los intervalos quedan conformados según se muestra en la Tabla 3:

Tabla 3. Estados de la Naturaleza y Rangos de la Variable IGBVL

\begin{tabular}{|c|c|}
\hline Estado & Rango \\
\hline 0 & Desde 1427.23 hasta 1443.99 puntos \\
\hline 1 & Desde 1443.99 hasta 1460.75 puntos \\
\hline 2 & Desde 1460.75 hasta 1477.50 puntos \\
\hline 3 & Desde 1477.50 hasta 1419.26 puntos \\
\hline 4 & Más de 1494.26 puntos \\
\hline
\end{tabular}

Considerando estos rangos, se revisó la estadística y se contabilizó las frecuencias para las diferentes combinaciones de transiciones desde un estado $i$ hasta un estado $j$, considerando que tanto $i$ como $j$ comprenden los valores 0, 1, 2, 3 y 4. Los resultados se muestran en la Tabla 4:

Tabla 4. Distribución de Frecuencias para la Transición de Estados

\begin{tabular}{|c|c|c|c|}
\hline $\begin{array}{c}\text { Transición } \\
\text { desde el } \\
\text { estado } \mathbf{i h a s t a} \\
\text { el estado } \mathbf{j}\end{array}$ & Frecuencia & $\begin{array}{c}\text { Transición } \\
\text { desde el } \\
\text { estado } \mathbf{i} \text { hasta } \\
\text { el estado } \mathbf{j}\end{array}$ & Frecuencia \\
\hline $0-0$ & 3 & $2-3$ & 5 \\
\hline $0-1$ & 3 & $3-2$ & 5 \\
\hline $1-0$ & 1 & $3-2$ & 0 \\
\hline $1-1$ & 16 & $3-3$ & 10 \\
\hline $1-2$ & 7 & $3-4$ & 5 \\
\hline $2-0$ & 1 & $4-3$ & 5 \\
\hline $2-1$ & 6 & $4-4$ & 8 \\
\hline $2-2$ & 28 & & \\
\hline Total & $103^{*}$ & & \\
\hline
\end{tabular}

*Se pierde un dato al calcular las transiciones debido al estado inicial de referencia 0.
De esta forma se obtiene la matriz de transiciones para este problema:

\section{Tabla 5. Matriz de Transiciones de los Estados de la Variable IGBVL}

\begin{tabular}{|c|c|c|c|c|c|}
\hline & 0 & 1 & 2 & 3 & 4 \\
\hline 0 & 0.5000 & 0.5000 & 0 & 0 & 0 \\
\hline 1 & 0.0417 & 0.6667 & 0.2917 & 0 & 0 \\
\hline 2 & 0.0250 & 0.1500 & 0.7000 & 0.1250 & 0 \\
\hline 3 & 0 & 0 & 0.2500 & 0.500 & 0.2500 \\
\hline
\end{tabular}

Nota. La información en cada celda es la probabilidad de ir desde elestado $i$ (fila) al estado $j$ (columna).

\section{CÁLCULO DE LAS PROBABILIDADES DE ESTADO ESTABLE}

Basándose en las ecuaciones de Chapman - Kolmogorov tenemos el siguiente sistema de ecuaciones para hallar las probabilidades de estado estable $\pi_{\mathrm{j}}$

$$
\begin{aligned}
& \pi_{0}=0.5 \pi_{0}+0.0417 \pi_{1}+0.0250 \pi_{2} \\
& \pi_{1}=0.5 \pi_{0}+0.6667 \pi_{1}+0.15 \pi_{2} \\
& \pi_{2}=0.2917 \pi_{1}+0.7 \pi_{2}+0.25 \pi_{3} \\
& \pi_{3}=0.1250 \pi_{2}+0.5 \pi_{3}+0.3846 \pi_{4} \\
& \pi_{4}=0.25 \pi_{3}+0.6154 \pi_{4} \\
& \pi_{0}+\pi_{1}+\pi_{2}+\pi_{3}+\pi_{4}=1
\end{aligned}
$$

Resolviendo el sistema de ecuaciones, compuesto por las ecuaciones (2) - (7) obtenemos las siguientes probabilidades de estado estable (ver Tabla 6):

\section{Tabla 6. Probabilidades de Estado Estable}

\begin{tabular}{l|c|}
\hline Estado & Probablidad \\
\hline $0\left(\pi_{0}\right)$ & 0.0396 \\
$1\left(\pi_{1}\right)$ & 0.2376 \\
$2\left(\pi_{2}\right)$ & 0.3960 \\
$3\left(\pi_{3}\right)$ & 0.1980 \\
$4\left(\pi_{4}\right)$ & 0.1287 \\
Total & 1.000 \\
& \\
PROYECCIÓN DEL FLUJO DE CAJA PARA LAS \\
ACCIONES SELECCIONADAS
\end{tabular}

Para obtener los flujos de caja se obtendrán los promedios de la variable IGBVL por cada estado y luego se obtendrá el precio por acción en función de las regresiones previamente calculadas, en términos de la ecuación (1) 
Tabla 7. Precios por Acción en Función del Estado de la Variable IGBVL

\begin{tabular}{|c|c|c|c|c|}
\hline \multirow{2}{*}{ Estado } & \multirow{2}{*}{$\begin{array}{c}\text { Valor } \\
\text { promedio } \\
\end{array}$} & \multicolumn{3}{|c|}{ Precios por acción } \\
\cline { 3 - 5 } & IGVL & ALICORC1 & CREDITC1 & BUENAVC1 \\
\hline 0 & 1435.61 & $\mathrm{~S} / 10.49$ & $\mathrm{~S} / 6.34$ & $\mathrm{~S} / 50.75$ \\
\hline 1 & 1452.37 & $\mathrm{~S} / 10.63$ & $\mathrm{~S} / 6.40$ & $\mathrm{~S} / 51.45$ \\
\hline 2 & 1469.13 & $\mathrm{~S} / 10.77$ & $\mathrm{~S} / 6.45$ & $\mathrm{~S} / 52.16$ \\
\hline 3 & 1485.88 & $\mathrm{~S} / 10.92$ & $\mathrm{~S} / 6.51$ & $\mathrm{~S} / 52.86$ \\
\hline 4 & 1502.64 & $\mathrm{~S} / 11.06$ & $\mathrm{~S} / 6.56$ & $\mathrm{~S} / 53.56$ \\
\hline
\end{tabular}

Con la proyección del precio de las acciones se presentan las ganancias (pérdidas) esperadas correspondientes, debido al cambio de los estados en la variable IGBVL. Los resultados se presentan en la Tabla 8:

Tabla 8. Flujo de Efectivo Esperado por Acción y por Estado de la Variable IGBVL (en soles)

\begin{tabular}{|c|c|c|c|}
\hline Estado & ALICORC1 & CREDITC1 & BUENAVC1 \\
\hline 0 & -0.0132 & -0.0050 & -0.0642 \\
\hline 1 & 0.0503 & 0.0192 & 0.2464 \\
\hline 2 & 0.0060 & 0.0023 & 0.0293 \\
\hline 3 & -0.0373 & -0.0143 & -0.1828 \\
\hline 4 & 0.0359 & 0.0137 & 0.1760 \\
\hline
\end{tabular}

Como ejemplo, el flujo de caja esperado para Alicorp, respecto al estado 0 , es el siguiente: de acuerdo con la matriz de transición, llegar el estado 0 se puede lograr de dos maneras, desde el estado $1\left(p_{10}\right)$ y desde el estado 2 $\left(p_{20}\right)$, con probabilidades 0.0417 y 0.0250 , respectivamente. Estos cambios de estado representan pérdidas, ya que son disminuciones del valor promedio de IGVBL y por consiguiente disminuciones en los precios de las acciones. Para ambas transiciones las pérdidas calculadas de acuerdo a la tabla 10 son S/ 0.14 y S/ 0.29 respectivamente. De esta forma el flujo de caja esperado se calcula de la siguiente manera:

$-0.14(0.0417)-0.29(0.0250)=-0.0132$

De acuerdo al proceso de decisión Markoviano (PDM) el rendimiento final esperado de una política de decisión se obtiene multiplicando el flujo de caja esperado de cada estado por la probabilidad de estado estable de dicho estado. Como ejemplo, si se tomara la decisión de sólo invertir en la acción de Alicorp, obtendríamos el siguiente rendimiento final de la política seleccionada:
Tabla 9. Rendimiento Esperado para la Política de Invertir sólo en Alicorp (ALICORC1)

\begin{tabular}{|c|c|c|}
\hline Estado & $\begin{array}{c}\text { Flujo de caja } \\
\text { esperado (en soles) }\end{array}$ & $\begin{array}{c}\text { Probabilidad de } \\
\text { estado estable }\end{array}$ \\
\hline 0 & -0.0132 & 0.0396 \\
\hline 1 & 0.0503 & 0.2376 \\
\hline 2 & 0.0060 & 0.3960 \\
\hline 3 & 0.0373 & 0.1980 \\
\hline 4 & 0.0359 & 0.1287 \\
\hline $\begin{array}{c}\text { Rendimiento } \\
\text { esperado }\end{array}$ & $\mathbf{0 . 0 1 1 0}$ & \\
\hline
\end{tabular}

¿Cuántas políticas se pueden identificar en este caso? Aquí se tienen varias opciones, pues por cada estado se puede tomar la decisión de invertir seleccionando entre tres acciones, pudiendo repetir la inversión en una acción para diferentes estados de la variable IGBVL. Se debe recurrir, por tanto, a las permutaciones con repeticiones; $\mathrm{y}$ considerando que existen 5 estados para la variable IGBVL y tres acciones para invertir, se obtiene la cantidad de 243 políticas de inversión. Para conocer la política óptima habría que calcular el rendimiento esperado de cada política y luego seleccionar aquella(s) que tenga(n) el valor más alto.

Conforme aumenta el número de estados y acciones para invertir, se puede deducir que las alternativas aumentan de manera considerable, dificultando el procedimiento de obtener una solución adecuada en un tiempo razonable. De lo anterior queda clara la utilidad de desarrollar un modelo de programación lineal (PL) para obtener directamente la política óptima de inversión, como se pretende en esta investigación y que se desarrolla en la siguiente sección.

\section{PROPUESTA DE MODELO DE PROGRAMACIÓN LINEAL}

El modelo propuesto, consistente con lo que comenta Hillier (2010), tiene la siguiente estructura: La variable de decisión es $\mathrm{y}_{i k^{\prime}}$ que es la probabilidad incondicional de que el sistema esté en el estado $i$ y se toma la decisión $k$. Cabe recordar que lo estados i se refieren a los valores que puede asumir el IGBVL, mientras que la decisión $k$ se refiere a la decisión de invertir o no en una determinada acción. En cuanto a la función objetivo, ésta debe buscar la maximización del flujo de efectivo esperado a largo plazo de las decisiones de inversión, por lo que planteamos:

$\operatorname{Max} Z=\sum_{i=0}^{M} \sum_{k=1}^{K} U_{i k} y_{i k}$ 
En cuanto a las restricciones, la suma de todas las probabilidades incondicionales debe ser uno, por lo que:

$\sum_{i=0}^{M} \sum_{k=1}^{K} y_{i k}=1$

La probabilidad de estado estable $\left(\pi_{i}\right)$ que se ha determinado previamente está relacionada con la variable $y_{i k^{\prime}}$, en el sentido de que si se suman todas las probabilidades incondicionales del estado $i$ que se derivan de todas las decisiones $k$ que se adoptan, llegaremos a la probabilidad de estado estable del sistema; de esta manera tenemos:

$$
\begin{aligned}
& \pi_{i}=\sum_{k=1}^{K} y_{i k} \\
& \sum_{k=1}^{K} y_{j k}=\sum_{i=0}^{M} \sum_{k=1}^{K} y_{i k} p_{i j}(k), \text { para } j=0,1, \ldots, M
\end{aligned}
$$

donde $p_{i j}(k)$ es la probabilidad de transición desde el estado $i$ al estado $j$, dado que se ha tomado la decisión $k$. Finalmente, se debe llegar a tomar una decisión, llámese $D_{i k^{\prime}}$, como la probabilidad de tomar la decisión $k$, dado que el sistema se encuentra en el estado i. La relación entre $D_{i k}$ y las variables de decisión $y_{i k}$ se obtiene a través de la teoría de la probabilidad condicional; y es la siguiente:

$y_{i k}=\pi_{i} D_{i k}$

$D_{i k}=\frac{y_{i k}}{\sum_{k=1}^{K} y_{i k}}$

De esta manera, es posible utilizar el modelo de programación lineal y resolverse a través del método Símplex. El complemento solver de MS Excel puede realizar dicha tarea y la interfaz preparada para este artículo es la siguiente:

\section{Figura 6. Interfaz del Modelo de Programación Lineal en MS Excel}

\section{Matriz de transición}

\begin{tabular}{|c|c|c|c|c|c|}
\hline Estados & $\mathbf{0}$ & $\mathbf{1}$ & $\mathbf{2}$ & $\mathbf{3}$ & $\mathbf{4}$ \\
\hline 0 & 0.5000 & 0.500 & 0 & 0 & 0 \\
\hline 1 & 0.0417 & 0.6667 & 0.2916 & 0 & 0 \\
\hline 2 & 0.0250 & 0.1500 & 0.7000 & 0.1250 & 0 \\
\hline 3 & 0 & 0 & 0.2500 & 0.5000 & 0.2500 \\
\hline 4 & 0 & 0 & 0 & 0.3846 & 0.6154 \\
\hline
\end{tabular}

\section{Matriz de flujo de efectivo}

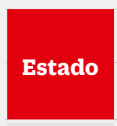

\section{Valor
promedio IGBVL}

\begin{tabular}{|l|l|l|}
\hline \multicolumn{3}{|c|}{ Precios por acción } \\
\hline ALICORC1 & CREDITC1 & BUENAVC1 \\
\hline
\end{tabular}

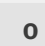

1435.61

$-S / 0.0132$

$-S / 0.0050$

$-S / 0.0645$

1

1452.37

S/ 0.0503

S/ 0.0192

S/ 0.2464

1469.13

$\mathrm{S} / 0.0060$

S/0.0023

S/ 0.0293

\begin{tabular}{l|l}
3 & 1485.88
\end{tabular}

$-S / 0.0373$

$-S / 0.0143$

S/ 0.1828

1502.64

S/ 0.0359
Matriz de decisiones y restricciones

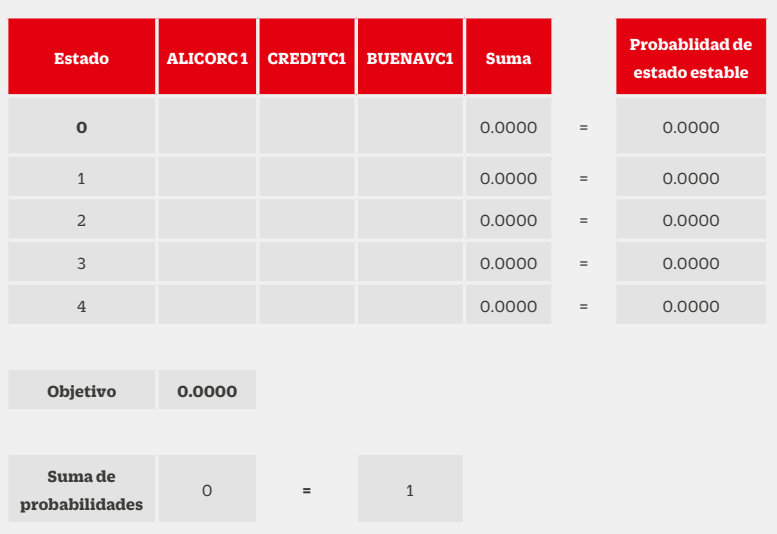

Aplicando el complemento Solver, a través del algoritmo Símplex, obtenemos el siguiente resultado:

\section{Figura 7. Resultado del Modelo de Programación Lineal}

Matriz de decisiones y restricciones

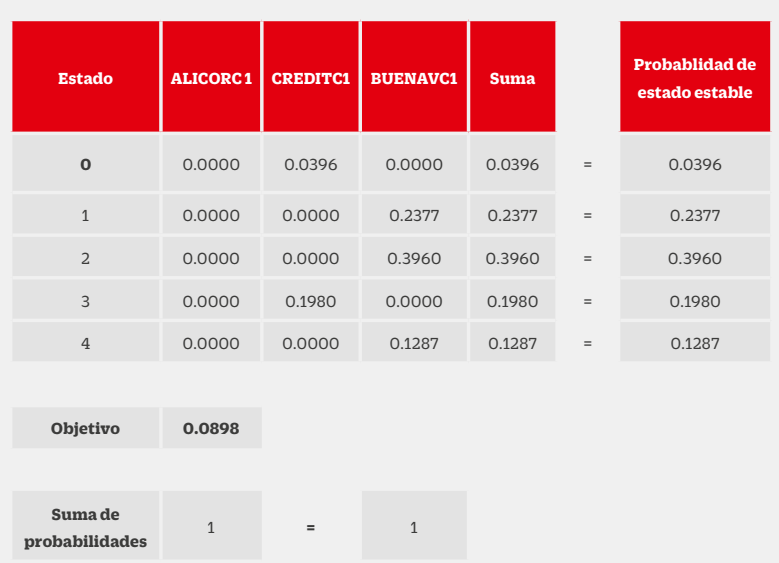

Nótese que en la figura del resultado del modelo, los valores de la columna "probabilidad de estado estable" coinciden con los valores previamente calculados en la tabla 6. Las celdas en color celeste corresponden a las variables $y_{i k}$ mencionadas en el planteamiento original; con estos resultados podemos obtener las decisiones $D_{i k^{\prime}}$ que se resumen en la siguiente tabla:

Tabla 10. Decisiones Óptimas

\begin{tabular}{|c|c|c|c|}
\hline Estado & ALICORC1 & CREDITC1 & BUENAVC1 \\
\hline 0 & 0 & 1 & 0 \\
\hline 1 & 0 & 0 & 1 \\
\hline 2 & 0 & 0 & 1 \\
\hline 3 & 0 & 1 & 0 \\
\hline 4 & 0 & 0 & 1 \\
\hline
\end{tabular}

Es así que las recomendaciones son las siguientes: 
Tabla 11. Acciones Correspondientes en Función del Estado de la Variable IGBVL

\begin{tabular}{|c|c|}
\hline Estado & Acción \\
\hline 0 & CREDITC1 \\
\hline 1 & BUENAVC1 \\
\hline 2 & BUENAVC1 \\
\hline 3 & CREDITC1 \\
\hline 4 & BUENAVC1 \\
\hline
\end{tabular}

De las 243 posibles políticas, ésta es la más adecuada. Comparando con la política de invertir sólo en ALICORC1 se obtiene un rendimiento aproximadamente ocho veces mayor. Es así que utilizando esta metodología es posible encontrar una política óptima de toma de decisiones.

\section{CONCLUSIONES}

Los procesos markovianos de decisión permiten modelar la toma de decisiones a través de la utilización de probabilidades. Dichas probabilidades se consideran de transición, debido a que la variable de interés se divide en estados a partir de los cuáles se desea saber la decisión óptima que se debe adoptar. Debido a que el número de posibles decisiones crece de manera importante conforme se incrementa el número de estados de la variable de interés, el uso de la enumeración exhaustiva de aquellas decisiones para alcanzar un óptimo se vuelve inmanejable. Una de las herramientas que se pueden utilizar para alcanzar una solución óptima en este caso es la programación lineal.

Para el caso analizado en el presente artículo, se revisó la información de tres activos financieros, representativos de diferentes industrias, que cotizan en la BVL. Con el cálculo de las correlaciones individuales, se pudo conocer el precio estimado de la acción respecto al estado de la variable que ocupa el Índice General de la BVL. El número de combiaciones respecto a la cantidad de activos a evaluar y el número de estados que puede tener el IGBVL asciende en este caso a 243, cifra que implicaría una revisión exhaustiva de cada política de inversión, y seleccionar aquellas que maximicen el beneficio de largo plazo.

Al desarrollar el modelo de programación lineal, se puede observar que la política óptima depende del estado en que se encuentre el IGBVL, y esta política consiste en invertir en la acción CREDITC1 cuando nos encontremos en los estados 0 y 3 , mientras que en el resto de estados es conveniente invertir en BUENAVC1. Llama la atención que no se haya seleccionado a la acción ALICORC1, pero se debe dejar estipulado que el modelo de programación no impuso restricciones respecto a alguna inversión mínima en cada acción, dejando libremente al algoritmo tomar una decisión óptima, y es por ello que no se consideró a la acción mencionada. El presente modelo puede ser ampliado a un número mucho mayor de acciones, y también a la definición de mayores estados para la variable IGBVL, convirtiéndose en una interesante herramienta de toma de decisiones en el ámbito financiero.
Siempre será posible aplicar una técnica de optimización para resolver un caso de toma de decisiones, y esta técnica, aunque forma parte del conjunto "clásico", mantiene una vigencia importante, posibilitando el desarrollo empresarial.

\section{REFERENCIAS}

Bolsa de Valores de Lima [BVL]. (2018, 30 de mayo). Página Institucional. Obtenido en https://www.bvl.com.pe/

Hillier, F. \& Lieberman, G. (2010). Introducción a la Investigación de Operaciones. Ciudad de México, México: Mc-Graw Hill Interamericana.

Taha, H. (2012). Investigación de Operaciones. Ciudad de México, México: Pearson Educación de México.

Wei, Q. (2016). Continuous-time Markov decisión processes with risk-sensitive finite-horizon cost criterion. Mathematical Methods of Operations Research, 84(3), 461-487. doi:10.1007/s00186-016-0550-4 